\title{
Twin-to-Twin Transfusion Syndrome in
}

\section{monochorionic, monoamniotic twin pregnancy with common umbilical cord insertion}

\author{
Filip P. Szkodziak@, Jaroslaw Krzyzanowski®, Tomasz Paszkowski®, \\ Slawomir Wozniak (D), Piotr R. Szkodziak (D)

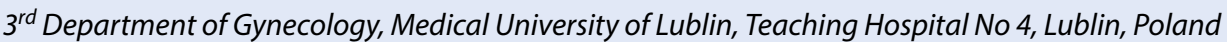

Key words: Twin-to-Twin Transfusion Syndrome; TTTS; monochorionic monoamniotic twin pregnancy; common umbilical cord insertion; multiple pregnancy

Ginekologia Polska 2022; 93, 2: 177-178

Twin-to-Twin Transfusion Syndrome, TTTS, monochorionic monoamniotic twin pregnancy, common umbilical cord insertion, multiple pregnancy. Initial clinical features of Twin-to-Twin Transfusion Syndrome (TTTS) result from discordance in the intravascular volume between twins. Monochorionic (MC) twins share a single placenta, and, in fact, all have some component of TTTS due to the presence of intertwin placental vascular anastomoses. However, most MC multiple pregnancies represent a balanced blood flow through the placental anastomoses, avoiding significant TTTS-specific consequences [1,2].

A 24-year-old patient at 28 weeks of MC, monoamniotic twin pregnancy was hospitalized due to decreased fetal movements of one twin. The patient received antenatal corticosteroid therapy at 26 weeks of pregnancy (betamethasone $2 \times 12 \mathrm{mg}$ intravenously with an interval of $24 \mathrm{~h}$ between doses). Fetal cardiac rhythm was regular, 150 beats per minute (bpm) in the presenting twin (twin A) and $170 \mathrm{bpm}$ in the non-presenting twin (twin B). Both fetuses were in a transverse lie. After 15 minutes of cardiotocography, a deceleration (up to $60 \mathrm{bpm}$ ) was noted in twin B. Fenoterol was administered according to the intrauterine resuscitation guidelines. Fetal well-being was assessed by ultrasound Doppler examination of the Umbilical Artery (UA), Umbilical Vein (UV) and Ductus Venosus (DV).

Twin A had an estimated fetal weight (EFW) of $1250 \mathrm{~g}$, corresponding to 28 weeks and 4 days. Blood flow in the UA, UV, and DV was normal with no signs of brain sparing effect.

Twin B had an EFW of $1030 \mathrm{~g}$, corresponding to 26 weeks and 1 day. Doppler examination revealed reversed end-diastolic flow in the UA, a pulsatile flow in the UV, and reverse a-wave flow in the DV with signs of brain sparing effect.

There was an excessive accumulation of amniotic fluid. Moreover, normal fetal activity and breathing in Twin A and abnormal fetal activity and breathing in twin B were found.

The patient qualified for an emergency cesarean section. Two girls, $1190 \mathrm{~g}$ and $1060 \mathrm{~g}$ were born. Typical signs of TTTS were confirmed by neonatologists. During the cesarean section, the MC monoamniotic pregnancy was confirmed. Examination of the placenta revealed a single, common, central umbilical cord insertion (single trunk). The umbilical cord of the donor was half the size of the recipient's one (Fig. 1).

The currently applied management options of TTTS include expectant management, amnioreduction (AR), serial amnioreduction (SAR), fetoscopic laser photocoagulation of placental anastomoses techniques (non-selective technique, selective laser photocoagulation, sequential selective laser photocoagulation, Solomon technique) and selective feticide [1-3].

During the literature review, we did not find any previous reports of TTTS in MC, monoamniotic twin pregnancy with common umbilical cord insertion. Such a case should be considered during diagnostics, as its treatment options are limited compared to TTTS with normal umbilical cord insertion. In the presented case, fetoscopic laser photocoagulation of placental anastomoses techniques could not be applied. First of all, TTTS was diagnosed at 28 weeks of pregnancy, whereas today, these techniques could be applied between 16 and 26 weeks. Furthermore, due to the common umbilical cord insertion, such a procedure was technically impossible to perform. AR and SAR are palliative management options of TTTS. In the presented case, an immediate intervention was required due to the deteriorating health of the fetuses. Single AR would not sufficiently improve that. Because of threatened asphyxia of one twin, an emergency cesarean section was the only available treatment option.

Corresponding author:

Piotr R. Szkodziak

Medical University of Lublin, 3rd Department of Gynecology, Teaching Hospital No 4, 20-954 Lublin, Poland

e-mail: piotr.szkodziak@gmail.com 


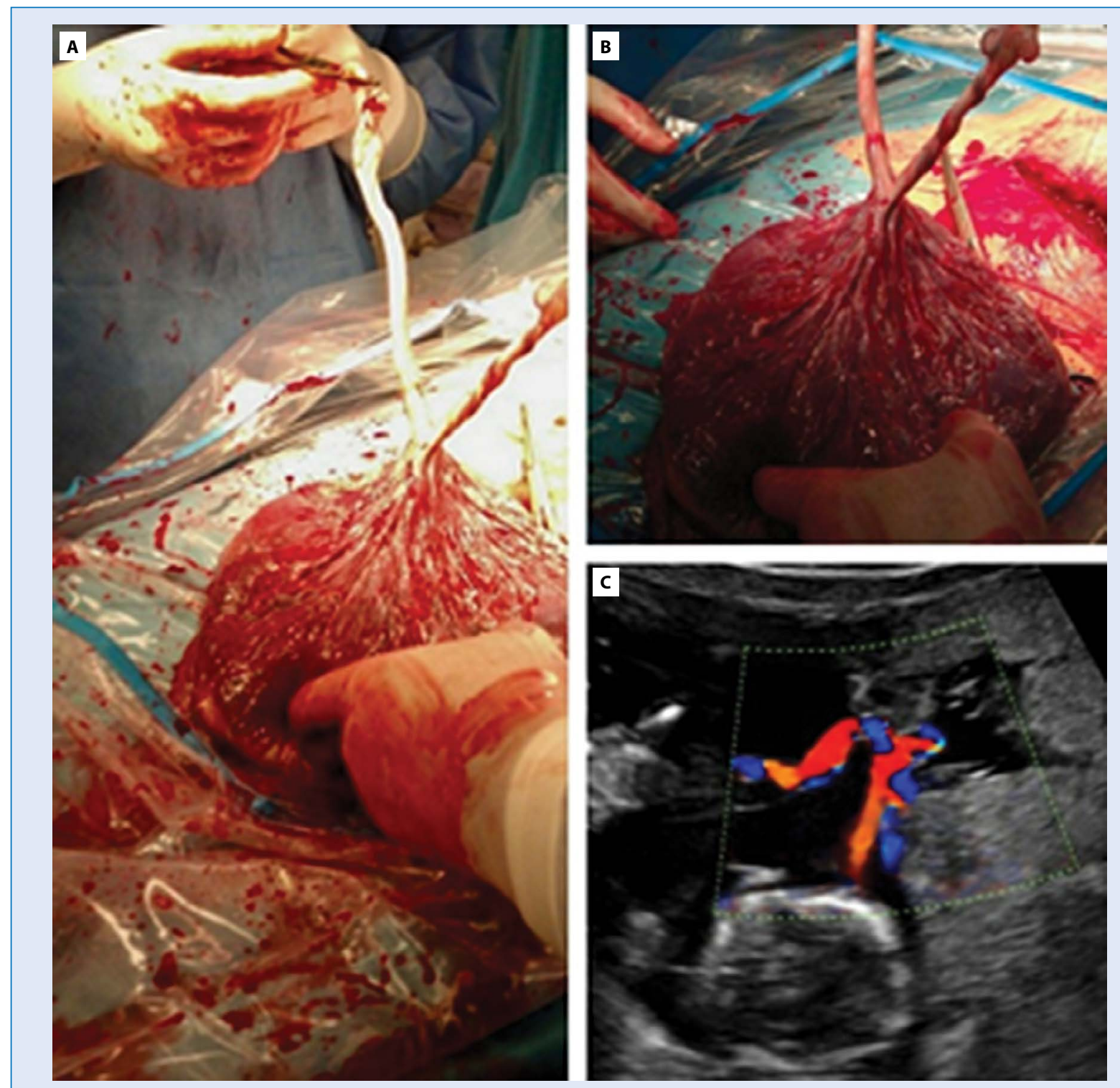

Figure 1. A-B. Common, central umbilical cord insertion (single trunk); C. Close proximity of umbilical cord insertions in twins at 12 weeks of pregnancy

\section{Funding}

Source of financing: funds allocated to statutory activities of the 3rd Chair and Department of Gynecology, Medical University of Lublin.

\section{Conflicts of interest}

The authors declare no conflict of interest.

\section{REFERENCES}

1. Johnson A. Diagnosis and Management of Twin-Twin Transfusion Syndrome. Clin Obstet Gynecol. 2015; 58(3): 611-631, doi: 10.1097/GRF.0000000000000128, indexed in Pubmed: 26165181.

2. Bamberg C, Hecher K. Update on twin-to-twin transfusion syndrome. Best Pract Res Clin Obstet Gynaecol. 2019; 58: 55-65, doi: 10.1016/j.bpobgyn.2018.12.011, indexed in Pubmed: 30850326.

3. Simpson L. Twin-twin transfusion syndrome. American Journal of Obstetrics and Gynecology. 2013; 208(1): 3-18, doi: 10.1016/j.ajog.2012.10.880. 